\title{
Unknown Inputs Nonlinear Observer for an Activated Sludge Process
}

\author{
Feten Smida (D), Taoufik Ladhari (D), Salim Hadj Saïd, and Faouzi M’sahli \\ Department of Electrical Engineering, ENIM, Road Ibn Eljazzar, 5019 Monastir, Tunisia \\ Correspondence should be addressed to Feten Smida; smida.feten@gmail.com
}

Received 6 March 2018; Revised 15 May 2018; Accepted 5 August 2018; Published 16 August 2018

Academic Editor: Olfa Boubaker

Copyright (C) 2018 Feten Smida et al. This is an open access article distributed under the Creative Commons Attribution License, which permits unrestricted use, distribution, and reproduction in any medium, provided the original work is properly cited.

\begin{abstract}
This paper deals with the jointly estimation problem of unknown inputs and nonmeasured states of one altering aerated activated sludge process (ASP). In order to provide accurate and economic concentration measures during aerobic and anoxic phases, a cascade high gain observer (HGO) approach is developed. Only two concentrations are available; the other process's states are assumed unavailable. The observer converges asymptotically and it leads to a good estimation of the unavailable states which are the ammonia and substrate concentration, as well as a quite reconstruction of the unknown inputs, which are the influent ammonia and the influent substrate concentrations. To highlight the efficiency of the proposed HGO with this MIMO system's dynamics, simulation results are validated with experimental data.
\end{abstract}

\section{Introduction}

The environmental conservation and the biological treatment of waste water are required to preserve the ecosystems. Therefore, modeling an activated sludge (AS) Waste Water Treatment Process (WWTP) has been one important area of research for three decades. This AS wastewater treatment process is physically, chemically, and biologically very complex. Furthermore the AS is nonlinear and unsteady system; this is caused by the flow rate variations in a waste water and its composition, joined to reactions varying in time within mingled microorganisms. In order to model the biological plant of the activated sludge process, a lot of models have been suggested: Activated Sludge Process Model N1 $(\operatorname{ASM}(1))$ in [1], the $\operatorname{ASM}(2)$ in [2], $\operatorname{ASM}(2 d)$ in [3], and $\operatorname{ASM}(3)$ in [4]. Due to the complexity of these models, various types of an activated sludge-plant reduced model have been put forward in the literature [5-10]. In [11] the authors presented one new model to alternate activated sludge models. In fact the mean continuous model would capture the switching dynamics governing behavior (i.e., aerobic-anaerobic phases), without any consideration of any switching behavior. Recently, [12] proposed a generalized state-identification classification modeling. As a matter of fact, image processing and analysis have been utilized on the basis of linear regression modeling.

More generally, waste water treatment processes have a few measurement and control equipment. Under these circumstances various models are utilized in controller design. Among control methods applied to the bioprocess we quote robust multimodel control using the Quantitative Feedback Theory (QFT) techniques in [13], adaptive control in [14, 15], and model predictive control (MPC) in $[16,17]$ and recently networked control in [18]. Nonetheless, the state variables of the ASM are not all available which present some difficulties in the eventual implementation.

In practice, for activated sludge process control and/or supervision, there are some nononline measured inputs, states, or concentrations (substrate concentration). Alternatively, these latter can also be hardly measured (ammonia and nitrate concentration): indeed the main difficulty towards the establishment of control strategies originates in general from the absence online, cheap, and reliable instrumentation. Even the existing sensors can be in many cases noisy and may require high maintenance and implementation cost. In the objective of solving such a problem, various observation methods are proposed. Among the observation method already studied we quote the following: [19] proposed for 
the nonlinear ASP model an extended Kalman filter, which works within the alternating aerobic-anoxic phase. The filter has been utilized for estimating states and nonstationary disturbances; [20] provided a brief summary of some results concerning state and parameter estimation approaches in relation to the chemical and biochemical processes. In [21] the authors resorted to a linear matrix inequality (LMI) to determine the values of the gain matrix of the asymptotic and classic observer, those are applied to a linearized bioprocess model. In the same framework a nonlinear observer which actually utilized both LMI and Lyapunov function was studied in [22]. In [11], the authors applied one classical Luenberger observer which was dedicated to continuous nonlinear models after linearization and it allows the estimation of nitrate and ammonia concentrations. Furthermore, to better developing the control strategies in presence of unknown inputs, several researches are devoted to such observation.

Studying dynamical systems when having UIs has highly given an incentive for research activities in state estimation, fault reconstruction, and control theory. By means of one Unknown Input Observer (UIO), simultaneously estimating the system state and the UIs is achievable. In [23], the authors extended the MIMO systems triangular class. In actual fact, these systems involved nonlinear inputs. In addition, they proposed one full order high gain observer in order to conjointly estimate nonmeasured state and UIs. The authors in [24] gave an essential and sufficient condition for UIO existence. In [25], the authors worked out UIO for one nonlinear system's canonical observable form in presence of uncertainties in LMI terms. The author in [26] designed a nonlinear differential algebraic systems' observation scheme with UI. The authors in [27] extended a standard high gain observer (HGO) with the aid of a sliding-mode-based term. This latter would follow the UI vector. Extending the work of $[28,29]$ designed a set of cascade high gain observers for many nonlinear MIMO systems. Each observer would provide the estimation of just an UI-vector component, with the exception of the last one which would really give an adjustment of full state variables. Given its utility, UIO has received big attention and has been applied to ASP, as in [30] where the authors suggested estimated states and the UIs as regards a minimized ASP nonlinear model through the use of the Extended Kalman Filter (EKF). Reference [31] treated the problems of Waste Water Treatment Plants (WWTP) sensor fault detection with the basic UIO (HGO). In fact, faults are just considered as unknown inputs. In a recent work, [32], an UIO is used to estimate jointly the states and an UI of the bioprocess linearized model.

The purpose of this paper is to estimate UIs and unavailable states of ASP. This contribution deals with the use of high gain UIO, which is relatively simple to design, can provide global or semiglobal stability results for a large class of nonlinear systems, robust to modeling uncertainty and external disturbances, and has a single adjustment parameter. The concentrations of ammonia and substrate in the inputs $\left(S_{\text {sin }}\right.$ and $\left.S_{\mathrm{NH}_{4} i n}\right)$ are very influential in the solution of the system model [33]. So the continuous varying inputs may seriously affect the system control; such problem can be solved by the use of an unknown inputs observer.

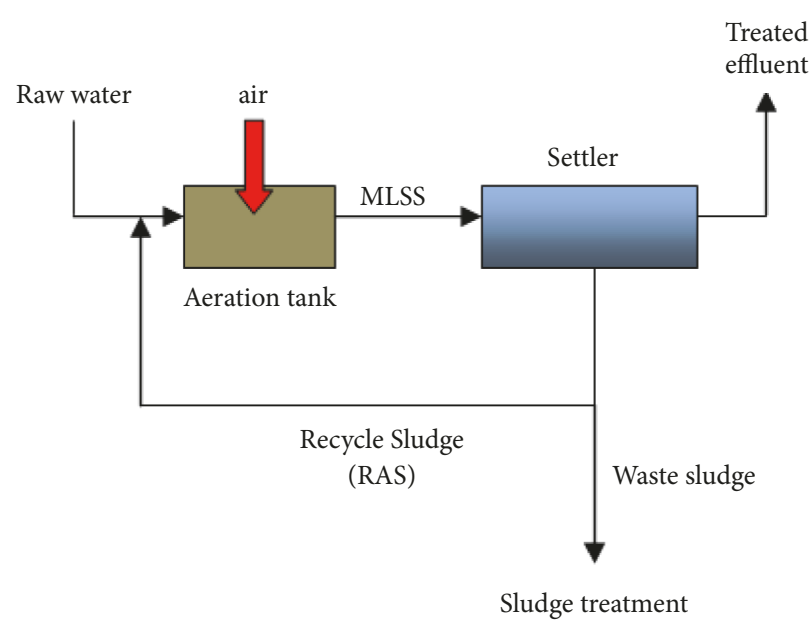

FIgURE 1: ASP diagram.

The paper is planned as follows. Initially we introduce a nonlinear ASP model. This model belongs to one specific nonlinear system class for which the development of a corresponding UIO is then detailed in section three; some preliminaries on the nonlinear systems class and state transformations are given in this section. Thereafter, in section four, and to facilitate applying the observation algorithm, we have proposed an appropriate repartition of ASP states. Simulation results are presented in the aim of highlighting the effectiveness of the proposed observer in curing one systematic routine so as to reconstruct jointly UIs and state variables for the ASP. A final conclusion ends the paper.

\section{Process Description}

There is a various model of ASP design. Precisely an ASP is composed of three principal components. The first component is an aeration tank. The latter will serve as a bioreactor. The second component is a settling tank (final clarifier). It was used to separate AS solids and treated waste water. The third one is a Return Activated Sludge (RAS) which is a tool for transferring settled AS from the clarifier into the aeration tank influent, (Figure 1). Added to that, the atmospheric air, or pure oxygen, will be put into a combination of primary treated sewage (waste water) which will be mixed with organisms in the purpose of developing a biological floc. In general, such a mixture is named as Mixed Liquor. Frequently, the concentration of the dry solids of mixed liquor suspended solid ranges between 3 and $6 \mathrm{~g} / \mathrm{L}$. Thereby, we control any removal efficiency by means of multiple operative conditions, for example, the aeration tank hydraulic residence time. To make it clear, the latter is determined by the division of the volume of the aeration tank over the flow rate. It is worth mentioning other factors like influent load (BOD5: Biological Oxygen Demand within 5 days, COD: Chemical Oxygen Demand, Nitrogen, etc.). The last factor is related to the AS solids which are existing in the aeration tank. We can mention here two other factors: oxygen supply and temperature. Mixed liquor will be evacuated, at the aeration 
tank discharge, into settling tanks. In addition to that the supernatant (treated waste water) ought to or should run off in the purpose of being discharged to a natural water. It can as well undergo further treatment before discharge. Because of biological growth, excess sludge has an eventual accumulation beyond the desired mixed liquor suspended solid concentration in the aeration tank. We remove such a solid amount from the treatment process in the target of keeping the biomass ratio to supplied food in balance. This solid amount, which is called the waste activated sludge, should be kept aside in storage tanks and should be further treated by digestion. This can be done prior to disposal under anaerobic or aerobic conditions.

2.1. Materiel and Method. The process considered in this work is the pilot ASWWTP unit in the Engineering Laboratory of Environmental Processes (ELEP) of the National Institution of Applied Sciences (NIAS) in Toulouse, France. It comprises a unique aeration tank with a volume $V_{a}=0.03 \mathrm{~m}^{3}$ equipped with an aeration surface that provides the necessary oxygen in the reactor in the goal of creating nitrification and denitrification conditions. It is obvious that the settler is a tank in which, in general, biomass is recirculated within the tank. In [34] mass balance was applied for each soluble (substrate) or particulate component (biomass). This led to an eleven state model that was subsequently reduced to four state variables. The earlier nonlinear models like ASM(1) were unattractive because of the high complex scheme systems. As a consequence, our focus is on reduced order models, which are on the bases of some biochemical considerations, giving the description of the nonlinear process behavior [33]. As a result, we distinguish aerobic and anoxic phases through the use of state variables associated with four substrate concentrations. As a matter of fact, aerobic phase is the aeration period. In the latter the air is injected within the reactor in big quantities in the objective of converting the pollution as ammonium nitrate. After that, in order to transform nitrate in nitrogen, in the anoxic phase, the aeration is stopped and also an optional extraneous carbon source is added inside the mixer. The operation of altering phases can be caused by the $k_{l a}$ oxygen transfer coefficient value which conspicuously varies from 0 (concerning the anoxic phase) to higher values (in what concerns the aerobic phase) (Figure 2).

2.2. System Model. The downsized system model is made up of four state variables which have just one alternating phase operation (alternating aeration). The latter is caused by the $k_{l a}$ oxygen transfer coefficient value changing from 0 to higher values. Tables 1 and 3 provide the process model's variable and parameters, while Table 2 gives the state variables. The process nonlinear model differential equations are expressed in the following [19]:

$$
\begin{aligned}
\dot{X}_{\mathrm{O}_{2}}= & -\left(D_{s}+D_{c}\right) X_{\mathrm{O}_{2}}+K_{L a}\left(S_{\mathrm{O}_{2 s a t}}-X_{\mathrm{O}_{2}}\right) \\
& -\frac{1-Y_{H}}{Y_{H}} \beta_{1}-4,57 \beta_{3} \\
\dot{X}_{N_{O_{3}}}= & -\left(D_{s}+D_{c}\right) X_{N_{3}}-\frac{1-Y_{H}}{2,86 Y_{H}} \beta_{2}+\beta_{3}
\end{aligned}
$$

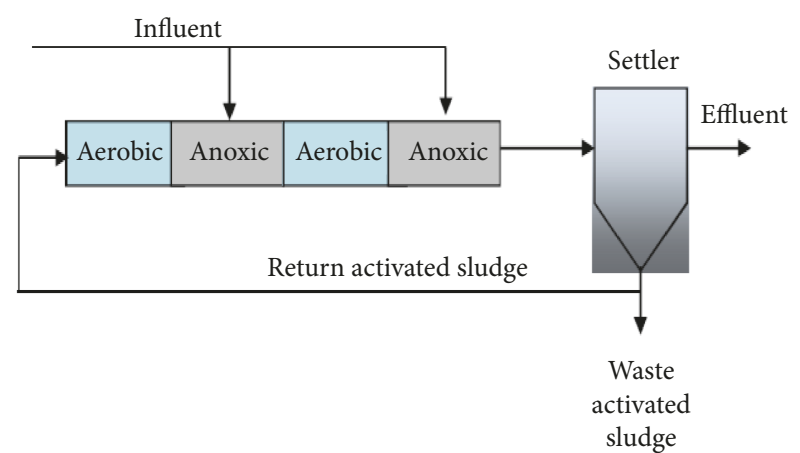

FIGURE 2: Combine aerobic and anoxic conditions to achieve nitrification and denitrification.

TABLE 1: Nonlinear-model parameters.

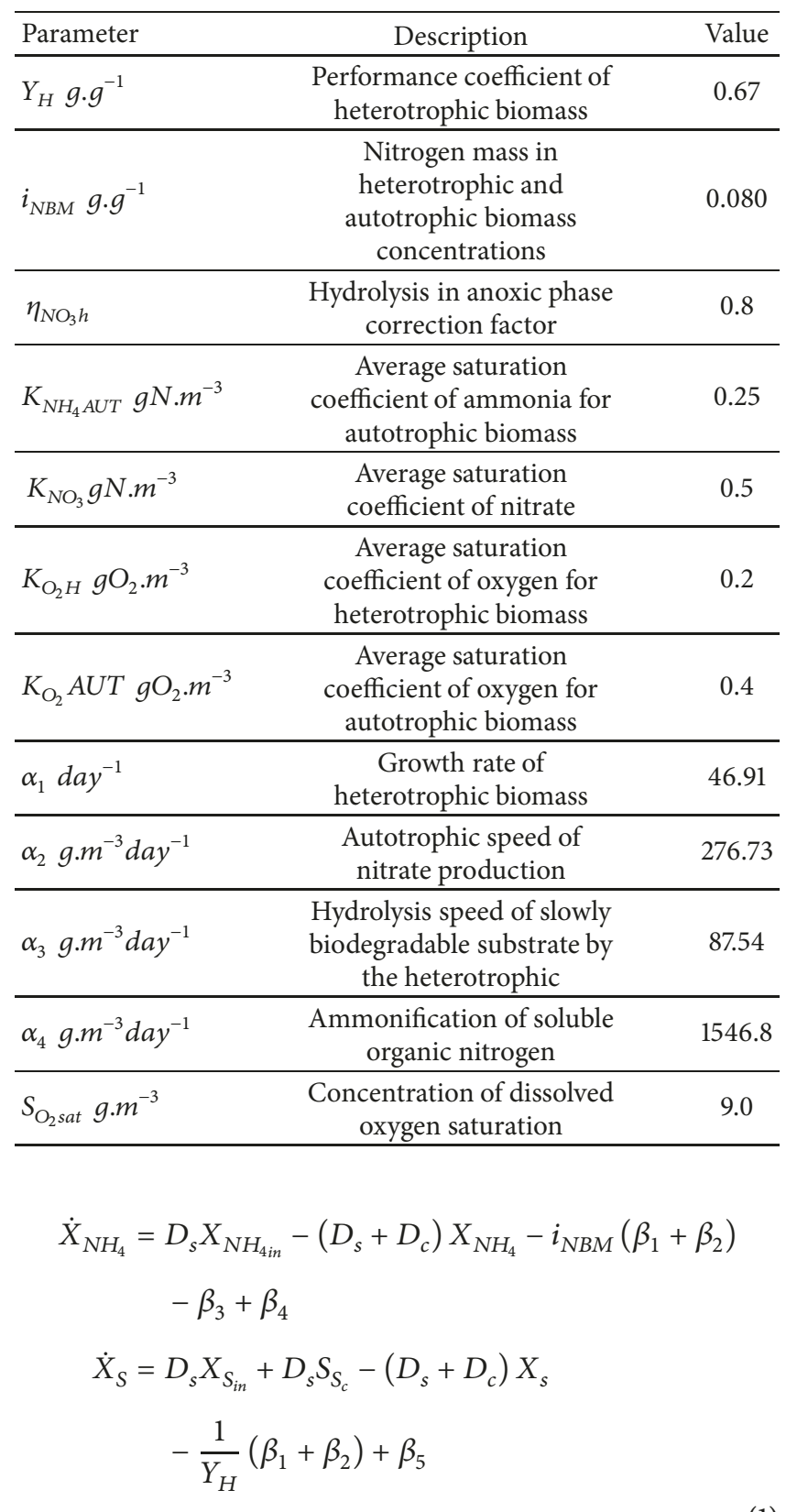


TABLE 2: State variables.

\begin{tabular}{lc}
\hline Notation & Description \\
\hline$X_{s}$ & Concentration of biodegradable substrate \\
$X_{\mathrm{NO}_{3}}$ & Concentration of nitrogen as nitrate and nitrite \\
$X_{\mathrm{NH}_{4}}$ & Concentration of nitrogen as ammonia \\
$X_{\mathrm{O}_{2}}$ & Concentration of oxygen \\
$X_{\mathrm{NH}_{4 i n}}$ & Concentration of ammonia input \\
$X_{\text {sin }}$ & Concentration of substrate input \\
\hline
\end{tabular}

TABLE 3: Input variables.

\begin{tabular}{lcc}
\hline Parameter & Description & Value \\
\hline$D_{s}$ & Input dilution rate & 2.58 day $^{-1}$ \\
$D_{c}$ & Dilution rate of external carbon source & $0.016 \mathrm{day}^{-1}$ \\
$S_{s c}$ & External carbon source's concentration & $16000 \mathrm{~g} \cdot \mathrm{m}^{-3}$ \\
$k_{L a}$ & Oxygen transfer coefficient & 215 or $0 \mathrm{day}^{-1}$ \\
\hline
\end{tabular}

with

$$
\begin{aligned}
& \beta_{1}=\alpha_{1} X_{s} \frac{X_{\mathrm{O}_{2}}}{X_{\mathrm{O}_{2}}+K_{\mathrm{O}_{2} \mathrm{H}}} \\
& \beta_{2}=\alpha_{1} X_{s} \frac{X_{\mathrm{NO}_{3}}}{X_{\mathrm{NO}_{3}}+K_{\mathrm{NO}_{3}}} \frac{K_{\mathrm{O}_{2} \mathrm{H}}}{X_{\mathrm{O}_{2}}+K_{\mathrm{O}_{2} \mathrm{H}}} \\
& \beta_{3}=\alpha_{2} \frac{X_{\mathrm{O}_{2}}}{X_{\mathrm{O}_{2}}+K_{\mathrm{O}_{2 a u t}}} \frac{X_{\mathrm{NH}_{4}}+K_{\mathrm{NH}_{4 a u t}}}{\beta_{4}=} \alpha_{3} \\
& \beta_{5}=\alpha_{4} \frac{X_{\mathrm{O}_{2}}}{X_{\mathrm{O}_{2}}+K_{\mathrm{O}_{2 a u t}}} \\
& +\eta_{\mathrm{NO}_{3} h} \frac{X_{\mathrm{NO}_{3}}}{X_{\mathrm{NO}_{3}}+K_{\mathrm{NO}_{3}}} \frac{K_{\mathrm{O}_{2} \mathrm{H}}}{X_{\mathrm{O}_{2}}+K_{\mathrm{O}_{2} \mathrm{H}}}
\end{aligned}
$$

$X_{\mathrm{O}_{2}}, X_{\mathrm{NO}_{3}}, X_{\mathrm{NH}_{4}}$, and $X_{s}$ are the concentrations of the dissolved oxygen, the nitrate, the ammonia, and the readily biodegradable substrate, respectively.

$X_{S_{i n}}$ is the concentration of substrate soluble in water and $X_{\mathrm{NH}_{4 i n}}$ is the concentration of the ammoniacal nitrogen entering the reactor. $X_{S_{i n}}$ and $X_{N H_{4 i n}}$ are considered the IUs of the system. $S_{S_{c}}$ is an oxygenous carbon source. $D_{s}$ and $D_{c}$ are the dilution rates determined as

$$
\begin{aligned}
& D_{s}=\frac{Q_{s}}{V_{a}}, \\
& D_{c}=\frac{Q_{c}}{V_{a}}
\end{aligned}
$$

where $Q_{s}$ is the input flow. In addition to that, $Q_{c}$ is an external carbon source flow.

$\beta_{i}$ (for $\mathrm{i}=1 . .5$ ) is a simpler form of the process kinetics $\rho_{i}$. It is directly linked to the standard mode ASM(1). Moreover, $\alpha_{i}$ is a specific parameter of the downsized nonlinear model.

\section{Studied Nonlinear Class and Design of Unknown Input Observer}

It is worth considering the nonlinear multioutput systems. These latter can be as follows:

$$
\begin{aligned}
& \dot{x}=f(u, x)+g(u, x, v)+\widetilde{\varepsilon}(t) \\
& y=x^{1}
\end{aligned}
$$

With $x=\left[\begin{array}{llll}x^{1^{T}} & x^{2^{T}} & \cdots & x^{q^{T}}\end{array}\right]^{T} \in \mathbb{R}^{n}$ where $x^{k} \in \mathbb{R}^{n_{k}}$ for $k=1,2, \ldots, q$ and $n_{1} \geq n_{2} \geq \cdots, n_{q}, \sum_{k=1}^{q} n_{k}=$ n. $\widetilde{\mathcal{E}}(t)=\left[0^{T}, \ldots, 0^{T}, \varepsilon^{q^{T}}\right]^{T}$ is an unknown function. The unknown input $v=v^{1} \in \mathbb{R}^{m}$, the known input $u \subset U$ a compact set of $\mathbb{R}^{u}$, and the output $y \in \mathbb{R}^{n_{1}}, f(u, x) \in \mathbb{R}^{n}$ with $f^{k}(u, x) \in \mathbb{R}^{n_{k}} . \lambda_{1}$ has been related to the unknown inputs. One notates $1 \leq \lambda_{1} \leq q$ such as

$$
\forall i<\lambda_{1}: \frac{\partial g^{i}}{\partial v^{1}}(u, x, v) \equiv 0,
$$

$$
\frac{\partial g^{\lambda_{1}}}{\partial v^{1}}(u, x, v) \neq 0
$$

System (1) is presented in its detailed form by the following equations:

$$
\begin{gathered}
\dot{x}^{1}=f^{1}\left(u, x^{1}, x^{2}\right)+g^{1}\left(u, x^{1}\right) \\
\vdots \\
\dot{x}^{\lambda_{1}-1}=f^{\lambda_{1}-1}\left(u, x^{1}, \ldots x^{\lambda_{1}}\right)+g^{\lambda_{1}-1}\left(u, x^{1}, \ldots x^{\lambda_{1}-1}\right) \\
\dot{x}^{\lambda_{1}}=f^{\lambda_{1}}\left(u, x^{1}, \ldots x^{\lambda_{1}+1}\right)+g^{\lambda_{1}}\left(u, x^{1}, \ldots x^{\lambda_{1}}, v^{1}\right) \\
\vdots \\
\dot{x}^{q-1}=f^{q-1}\left(u, x^{1}, \ldots x^{q}\right)+g^{q-1}\left(u, x^{1}, \ldots x^{q-1}, v^{1}\right) \\
\dot{x}^{q}=f^{q}\left(u, x^{1}, \ldots x^{q}\right)+g^{q}\left(u, x^{1}, x^{2}, \ldots x^{q}, v^{1}\right)+\varepsilon^{q} \\
y=x^{1}
\end{gathered}
$$

Note that this nonlinear system class is more general than those studied in $[23,28,35]$.

Let

$$
\varphi^{k}\left(u, x, v^{1}\right)=f^{k}(u, x)+g^{k}(u, x, v)+\widetilde{\varepsilon}(t)
$$

Our aim is to synthesize an observer to estimate at the same time the nonmeasured states and the vector of unknown inputs. In the target of dealing with the HGO synthesis, we make what follows:

(A.1) For $1 \leq k \leq q-1$ the function $x^{k+1} \longmapsto$ $f^{k}\left(u, x^{1}, \ldots, x^{k}, x^{k+1}\right)$ from $\mathbb{R}^{n_{k+1}} \longmapsto \mathbb{R}^{n_{k}}$ is injective for all $\left(u, x^{1}, \ldots, x^{k}, x^{k+1}\right)$. Then, there exist $\alpha_{1}, \beta_{1}>0$ such that $\forall k \in\{1, \ldots, q-1\}, \forall x \in \mathbb{R}^{n}, \forall u \in U$

$$
0<\alpha_{1}^{2} I_{n_{k+1}} \leq\left(\frac{\partial f^{k}}{\partial x^{k+1}}(u, x)\right)^{T} \frac{\partial f^{k}}{\partial x^{k+1}}(u, x) \leq \beta_{1}^{2} I_{n_{k+1}}
$$


where $I_{n_{k+1}}$ is a matrix identity, whose dimension is $\left(n_{k+1}\right) \times$ $\left(n_{k+1}\right)$.

Assumption (A1) is essential for guaranteeing the diffeomorphism existence which could make an initial system in the canonical form of observability.

(A.2) The function $\left(\begin{array}{c}x^{\lambda_{1}+1} \\ v^{1}\end{array}\right) \longmapsto \varphi^{\lambda_{1}}\left(u, x^{1}, \ldots, x^{\lambda_{1}}, x^{\lambda_{1}+1}\right.$, $\left.v^{1}\right)$ from $\mathbb{R}^{n_{\lambda_{1}+1}+m} \longrightarrow \mathbb{R}^{n_{\lambda_{1}}}$ is injective for all $\left(u, x^{1}, \ldots, x^{\lambda_{1}}\right.$, $\left.v^{1}\right)$; we consider

$$
F^{\lambda_{1}}\left(x, u, v^{1}\right)=\left(\frac{\partial \varphi^{\lambda_{1}}}{\partial x^{\beta_{1}+1}}\left(u, x, v^{1}\right) \frac{\partial \varphi^{\lambda_{1}}}{\partial v^{1}}\left(u, x, v^{1}\right)\right)
$$

Such assumption is a rank condition, which has to be opted for to estimate UIs. UIO:

Consequently, the next inequality is needed to design

$$
n_{\lambda_{1}+1}+m \leq n_{\lambda_{1}}
$$

In particular, we need to have

$$
m \leq p-1
$$

3.1. State Transformation. Now, a set of coordinate transformations should be introduced so as to canonically form system (4) corresponding to the HGO synthesis. Just as it should be, an augmented system is made up of 2 blocks such that their outputs have the same values as those of the original system (4). $x^{1}, \ldots, x^{\lambda_{1}+1}$ and $v^{1}$ are related first-block variables. On the other hand, variables linked to the last one should be able to be simple copies of the full system states.
As a consequence, we interpret the studied immersion like an injective map $\psi$ which is defined as follows:

$$
\begin{gathered}
\psi: \mathbb{R}^{n+m} \longrightarrow \mathbb{R}^{n_{1}+\cdots+n_{\lambda_{1}+1}+m+n} \text { and } \\
\left(\begin{array}{c}
x \\
v^{1}
\end{array}\right) \longmapsto \mu=\psi(x, v)=\left(\begin{array}{c}
\mu^{1} \\
\mu^{2}
\end{array}\right) \text {, with: } \\
\mu^{1}=\left(\begin{array}{c}
\mu_{1}^{1} \\
\mu_{2}^{1} \\
\vdots \\
\mu_{\lambda_{1}+1}^{1}
\end{array}\right) \in \mathbb{R}^{n_{1}+\cdots+n_{\lambda_{1}+1}+m},
\end{gathered}
$$

$$
\mu^{2}=\left(\begin{array}{c}
\mu_{1}^{2} \\
\mu_{2}^{2} \\
\vdots \\
\mu_{q}^{2}
\end{array}\right) \in \mathbb{R}^{n}
$$

$$
\psi(x, v):\left\{\begin{array}{l}
\mu_{k}^{1}=x^{k}, k=1, \ldots, \lambda_{1} \\
\mu_{\lambda_{1}+1}^{1}=\left(\begin{array}{c}
\mu_{\lambda_{1}+1,1}^{1} \\
\mu_{\lambda_{1}+1,2}^{i}
\end{array}\right)=\left(\begin{array}{c}
x^{\lambda_{1}+1} \\
v^{1}
\end{array}\right) \\
\mu_{k}^{2}=x^{k}, k=1, \ldots, q
\end{array}\right.
$$

Thus, the two subsystems in the $\mu$ coordinates are given in the following:

$$
\begin{gathered}
\dot{\mu}_{1}^{1}=f^{1}\left(u, \mu_{1}^{1}, \mu_{2}^{1}\right)+g^{1}\left(u, \mu_{1}^{1}\right) \\
\vdots \\
\dot{\mu}_{\lambda_{1}}^{1}=f^{\lambda_{1}}\left(u, \mu_{1}^{1}, \mu_{2}^{1}, \ldots, \mu_{\lambda_{1+1,1}}^{1}\right)+g\left(u, \mu_{1}^{1}, \mu_{2}^{1}, \ldots, \mu_{\lambda_{1}}^{1}, \mu_{\lambda_{1+1,2}}^{1}\right) \\
\dot{\mu}_{\lambda_{1}+1}^{1}=\left(\begin{array}{c}
f^{\lambda_{1}+1}\left(u, \mu_{1}^{1}, \mu_{2}^{1}, \ldots, \mu_{\lambda_{1+1,1}}^{1}, \mu_{\lambda_{1+2}}^{1}\right)+g\left(u, \mu_{1}^{1}, \mu_{2}^{1}, \ldots, \mu_{\lambda_{1+1,1}}^{1}, \mu_{\lambda_{1+1,2}}^{1}\right) \\
\varepsilon^{1}(t) \\
y_{1}=\mu_{1}^{1} \\
\dot{\mu}_{1}^{2}=f^{1}\left(u, \mu_{1}^{2}, \mu_{2}^{2}\right)+g^{1}\left(u, \mu_{1}^{2}\right) \\
\vdots \\
\dot{\mu}_{q-1}^{2}=f^{q-1}\left(u, \mu_{1: q}^{2}\right)+g^{q-1}\left(u, \mu_{1: q-1}^{2}, \mu_{\lambda_{1+2}}^{2}\right) \\
\dot{\mu}_{q}^{2}=f^{q}\left(u, \mu_{1: q}^{2}\right)+g^{q-1}\left(u, \mu_{1: q}^{2}, \mu_{\lambda_{1+2}}^{2}\right)+\varepsilon^{q} \\
y_{2}=\mu_{1}^{2}
\end{array}\right.
\end{gathered}
$$

with $\varepsilon^{1}(t)$ is an unknown bounded function. 
In order to obtain the canonical form, a second injective map $\phi$ is introduced as follows: $\Phi: \mathbb{R}^{n_{1}+\cdots+n_{\lambda_{1}+1}+m+n} \longrightarrow$ $\mathbb{R}^{p\left(\lambda_{1}+1\right) p q}$

$$
\left(\begin{array}{l}
\mu^{1} \\
\mu^{2}
\end{array}\right) \longmapsto \zeta=\Phi(u, \mu)=\left(\begin{array}{l}
\zeta^{1}=\Phi_{1}(u, \mu) \\
\zeta^{2}=\Phi_{2}(u, \mu)
\end{array}\right)
$$

with

$$
\begin{gathered}
\zeta^{1}=\left(\begin{array}{c}
\zeta_{1}^{1} \\
\zeta_{2}^{1} \\
\vdots \\
\zeta_{\lambda_{1}+1}^{1}
\end{array}\right) \in \mathbb{R}^{\left(\lambda_{1}+1\right) p} \\
\zeta^{2}=\left(\begin{array}{c}
\zeta_{1}^{2} \\
\zeta_{2}^{2} \\
\vdots \\
\zeta_{q}^{2}
\end{array}\right) \in \mathbb{R}^{q p}
\end{gathered}
$$

$\zeta_{j}^{1}$ and $\zeta_{j}^{2}$ are defined such as

$$
\begin{aligned}
& \zeta_{1}^{1}= \Phi_{1,1}\left(u, \mu_{1}^{1}\right)=\mu_{1}^{1} \\
& \zeta_{2}^{1}= \Phi_{1,2}\left(u, \mu_{1}^{1}, \mu_{2}^{1}\right)=f^{1}\left(u, \mu_{1}^{1}, \mu_{2}^{1}\right) \\
& \zeta_{3}^{1}= \Phi_{1,3}\left(u, \mu_{1}^{1}, \mu_{2}^{1}, \mu_{3}^{1}\right)=\frac{\partial f^{1}}{\partial \mu_{2}^{1}}\left(u, \mu_{1}^{1}, \mu_{2}^{1}\right) f^{1}\left(u, \mu_{1: 3}^{1}\right) \\
& \vdots \\
& \zeta_{\lambda_{1}}^{1}= \Phi_{1, \lambda_{1}}\left(u, \mu_{1: \lambda_{1}}^{1}\right)=\left(\prod_{k=1}^{\lambda_{1}-2} \frac{\partial f^{k}}{\partial \mu_{k+1}^{1}}\left(u, \mu_{1: k+1}^{1}\right)\right) \\
& \cdot f^{\lambda_{1}-1}\left(u, \mu_{1: \lambda_{1}}^{1}\right) \\
& \zeta_{\lambda_{1}+1}^{1}=\Phi_{1, \lambda_{1}+1}\left(u, \mu_{1: \lambda_{1}+1}^{1}\right) \\
&=\left(\prod_{k=1}^{\lambda_{1}-1} \frac{\partial f^{k}}{\partial \mu_{k+1}^{1}}\left(u, \mu_{1: k+1}^{1}\right)\right) \\
& \cdot\left(f^{\lambda_{1}}\left(u, \mu_{1: \lambda_{1}}^{1}, \mu_{\lambda_{1}+1,1}^{1}\right)+g^{\lambda_{1}}\left(u, \mu_{1: \lambda_{1}}^{1}, \mu_{\lambda_{1}+1,2}^{1}\right)\right) \\
& \zeta_{1}^{2}=\Phi_{2,1}\left(u, \mu_{1}^{2}\right)=\mu_{1}^{2} \\
& \zeta_{2}^{2}=\Phi_{2,2}\left(u, \mu_{1}^{2}, \mu_{2}^{2}\right)=f^{1}\left(u, \mu_{1}^{2}, \mu_{2}^{2}\right) \\
& \zeta_{3}^{2}=\Phi_{2,3}\left(u, \mu_{1}^{2}, \mu_{2}^{2}, \mu_{3}^{2}\right)=\frac{\partial f^{1}}{\partial \mu_{2}^{2}}\left(u, \mu_{1}^{2}, \mu_{2}^{2}\right) \\
& \cdot f^{1}\left(u, \mu_{1}^{2}, \mu_{2}^{2}, \mu_{3}^{2}\right)
\end{aligned}
$$

Using the adopted notation, system (12) can be written in the new coordinates $\zeta$ as follows:

$$
\begin{aligned}
& \dot{\zeta}_{1}^{1}=\zeta_{2}^{1} \\
& \dot{\zeta}_{2}^{1}=\zeta_{3}^{1}+\psi_{2}^{1}\left(u, \dot{u}, \zeta_{1}^{1}, \zeta_{2}^{1}\right)
\end{aligned}
$$

$$
\begin{aligned}
& \dot{\zeta}_{\lambda_{1}}^{1}=\zeta_{\lambda_{1}+1}^{1}+\psi_{\lambda_{1}}^{1}\left(u, \dot{u}, \zeta_{1}^{1}, \ldots, \zeta_{\lambda_{1}}^{1}\right) \\
& \dot{\zeta}_{\beta_{1}+1}^{1}=\psi_{\beta_{1}+1}^{1}(u, \dot{u}, \zeta) \\
& \quad+\left(\prod_{j=1}^{\lambda_{1}-1} \frac{\partial f^{j}}{\partial \mu_{j+1}^{1}}\left(u, \zeta^{i}\right)\right) \frac{\partial g^{\lambda_{1}}}{\partial v^{1}}\left(u, \zeta^{1}\right) \varepsilon(t) \\
& y_{1}=\zeta_{1}^{1} \\
& \dot{\zeta}_{1}^{2}=\zeta_{2}^{2} \\
& \dot{\zeta}_{2}^{2}=\zeta_{3}^{2}+\psi_{2}^{2}\left(u, \dot{u}, \zeta_{1}^{2}, \zeta_{2}^{2}\right) \\
& \dot{\zeta}_{\lambda_{1}}^{2}=\zeta_{\lambda_{1}+1}^{2}+\psi_{\lambda_{1}}^{2}\left(u, \dot{u}, \zeta_{1}^{2}, \ldots, \zeta_{\lambda_{i}}^{2}, \zeta^{1}\right) \\
& \dot{\zeta}_{\lambda_{1}+1}^{2}=\zeta_{\lambda_{1}+2}^{2}+\psi_{\lambda_{1}+1}^{2}\left(u, \dot{u}, \zeta_{1}^{2}, \ldots, \zeta_{\lambda_{1}}^{2}, \zeta_{\lambda_{1}+1}^{2}, \zeta^{1}\right) \\
& \vdots \\
& \dot{\zeta}_{q-1}^{2}=\zeta_{q-1}^{2}+\psi_{q-1}^{2}\left(u, \dot{u}, \zeta_{1}^{2}, \ldots, \zeta_{q-1}^{2}, \zeta^{1}\right) \\
& \dot{\zeta}_{q}^{2}=\psi_{q}^{2}(u, \dot{u}, \zeta) \varepsilon^{q} \\
& y_{2}=\zeta_{1}^{2}
\end{aligned}
$$

with 


$$
\begin{aligned}
\psi_{\lambda_{1}+1}^{1}(u, \dot{u}, \zeta)= & \frac{\partial \Phi_{1, \lambda_{1}+1}}{\partial u}\left(u, \mu_{1}^{1}, \ldots, \mu_{\lambda_{1}}^{1}, \mu_{\lambda_{1}+2}^{1}\right) \dot{u} \\
& +\sum_{j=1}^{\lambda_{1}}\left(\frac{\partial \Phi_{1, \lambda_{1}+1}}{\partial \mu_{j}^{1}}\left(u, \mu_{1}^{1}, \ldots, \mu_{\lambda_{1}+2}^{1}\right)\left(f^{k}\left(u, \mu_{1}^{1}, \ldots, \mu_{j+1}^{1}\right)+g^{k}\left(u, \mu_{1}^{1}, \ldots, \mu_{j}^{1}\right)\right)\right) \\
& +\frac{\partial \Phi_{1, \lambda_{1}+1}}{\partial \mu_{\lambda_{1}+1}^{1}}\left(u, \mu_{1}^{1}, \ldots, \mu_{\lambda_{1}+2}^{1}\right)\left(f^{\lambda_{1}+1}\left(u, \mu_{1}^{1}, \ldots, \mu_{\lambda_{1}+1}^{1}, \mu_{\lambda_{1}+2}^{1}\right)+g^{\lambda_{1}+1}\left(u, \mu_{1}^{1}, \ldots, \mu_{\lambda_{1}+2}^{1}\right)\right)
\end{aligned}
$$

and

$$
\begin{aligned}
\psi_{k}^{2}\left(u, \dot{u}, \zeta_{1}^{2}, \ldots, \zeta_{k}^{2}\right)= & \frac{\partial \Phi_{2, k}}{\partial u}\left(u, \mu_{1}^{2}, \ldots, \mu_{k}^{2}\right) \dot{u}+\left(\prod_{j=1}^{k-1} \frac{\partial f^{j}}{\partial \mu_{j+1}^{2}}\left(u, \mu_{1}^{2}, \ldots, \mu_{j+1}^{2}\right)\right) g^{k}\left(u, \mu_{1}^{2}, \ldots, \mu_{k}^{2}\right) \\
& +\sum_{j=1}^{k-1}\left(\frac{\partial \Phi_{2, k}}{\partial \mu_{j}^{2}}\left(u, \mu_{1}^{2}, \ldots, \mu_{k}^{2}\right)\left(f^{j}\left(u, \mu_{1}^{2}, \ldots, \mu_{j+1}^{2}\right)+g^{j}\left(u, \mu_{1}^{2}, \ldots, \mu_{j}^{2}\right)\right)\right)
\end{aligned}
$$

3.2. Observer Design. Our purpose is to synthesize an observer to estimate at the same time the vector of UIs as well as the nonmeasured state without the assumption of whatever model for UIs. According to Farza et al. (2004), a coordinate transformation is necessary for synthesizing such an observer. This may make one appropriate system form to synthesize the observer. Hence we should write systems (20)(21) as follows:

$$
\begin{aligned}
& \dot{\zeta}^{1}=A_{1} \zeta^{1}+\psi^{1}(\dot{u}, u, \zeta)+\bar{\varepsilon}^{1} \\
& y_{1}=\zeta_{1}^{1} \\
& \dot{\zeta}^{2}=A_{2} \zeta^{2}+\psi^{2}(\dot{u}, u, \zeta)+\bar{\varepsilon}^{2} \\
& y_{2}=\zeta_{1}^{2} \\
& A_{1}=\left(\begin{array}{cc}
0 & I_{\left(\lambda_{1}+1\right) p} \\
0 & 0
\end{array}\right) \\
& A_{2}=\left(\begin{array}{cc}
0 & I_{q p} \\
0 & 0
\end{array}\right)
\end{aligned}
$$

with

$$
\bar{\varepsilon}^{1}=\left[0_{\lambda_{1} n_{1}}^{T}, \ldots, \varepsilon^{1^{T}}\right]^{T}
$$

with

$$
\varepsilon^{1}=\left(\prod_{j=1}^{\lambda_{1}-1} \frac{\partial f^{j}}{\partial \mu_{j+1}^{1}}\left(u, \zeta^{i}\right)\right) \frac{\partial g^{\lambda_{1}}}{\partial v^{1}}\left(u, \zeta^{1}\right) \varepsilon
$$

and

$$
\bar{\varepsilon}^{2}=\psi_{q}^{2}(u, \dot{u}, \zeta) \varepsilon^{q}
$$

By referring to the framework given in [28], we opt for HGO synthesized for nonlinear MIMO systems, which are canonically nontriangular. The observer of systems (24)-(25) is designed as follows:

$$
\begin{aligned}
& \dot{\vec{\zeta}}^{1}=A_{1} \widehat{\zeta}^{1}+\psi^{1}(.)-\theta^{\delta_{1}} \Delta_{1}{ }^{-1}(\theta) S_{1}{ }^{-1} C_{1}^{T}\left(\widehat{\zeta}_{1}^{1}-\zeta_{1}^{1}\right) \\
& \dot{\vec{\zeta}}^{2}=A_{2} \hat{\zeta}^{2}+\psi^{2}(.)-\theta^{\delta_{2}} \Delta_{2}{ }^{-1}(\theta) S_{2}{ }^{-1} C_{2}^{T}\left(\hat{\zeta}_{1}^{2}-\zeta_{1}^{2}\right)
\end{aligned}
$$

with

$$
S_{1}{ }^{-1} C_{1}^{T}=\left[\begin{array}{c}
C_{\lambda_{1}+1}^{1} I_{p} \\
C_{\lambda_{1}+1}^{2} I_{p} \\
\vdots \\
C_{\lambda_{1}+1}^{\lambda_{1}+1} I_{p}
\end{array}\right] \text {, }
$$$$
S_{2}{ }^{-1} C_{2}^{T}=\left[\begin{array}{c}
C_{q}^{1} I_{p} \\
C_{q}^{2} I_{p} \\
\vdots \\
C_{q}^{q} I_{p}
\end{array}\right]
$$

$$
\begin{aligned}
& \Delta_{1}(\theta)=\operatorname{diag}\left(I_{p}, \frac{1}{\theta^{\delta_{1}}} I_{p}, \ldots, \frac{1}{\theta^{\lambda_{1} \delta_{1}}} I_{p}\right) \\
& \Delta_{2}(\theta)=\operatorname{diag}\left(I_{p}, \frac{1}{\theta} I_{p}, \ldots, \frac{1}{\theta^{q-1}} I_{p}\right)
\end{aligned}
$$


$\theta>0$ is a real number that represent just one observer design parameter. $\delta_{1}=2 q-3 ; \delta_{2}=1$.

Converging the estimation error dynamic was demonstrated in detail in [28].

This observer can also be given in the $\mu$ coordinates as

$$
\begin{aligned}
\dot{\hat{\mu}}^{1}= & f_{1}(u, \underline{\hat{\mu}})+g_{1}(u, \underline{\hat{\mu}}) \\
& -\left(\Lambda_{1}(u, \underline{\hat{\mu}})\right)^{+} \theta^{\delta_{1}} \Delta_{1}^{-1}(\theta) S_{1}^{-1} C_{1}^{T}\left(\widehat{\mu}_{1}^{1}-\mu_{1}^{1}\right) \\
\dot{\hat{\mu}}^{2}= & f_{2}(u, \underline{\hat{\mu}})+g_{2}(u, \underline{\hat{\mu}}) \\
& -\left(\Lambda_{2}(u, \underline{\hat{\mu}})\right)^{+} \theta^{\delta_{2}} \Delta_{2}^{-1}(\theta) S_{2}^{-1} C_{2}^{T}\left(\widehat{\mu}_{1}^{2}-\mu_{1}^{2}\right)
\end{aligned}
$$

Now, we suppose

$$
\begin{aligned}
\hat{x}^{k} & =\widehat{\mu}_{k}^{2} \quad \text { for } k=1, \ldots, q \\
\widehat{\eta}^{k} & =\widehat{\mu}_{k}^{1} \quad \text { for } k=1, \ldots, \lambda_{1} \\
\widehat{\eta}^{\lambda_{1}+1} & =\widehat{\mu}_{\lambda_{1}+1,1}^{1} \\
\hat{v}^{1} & =\widehat{\mu}_{\lambda_{1}+1,2}^{1}
\end{aligned}
$$

Utilizing this notation, we can express the observer as follows:

$$
\begin{aligned}
& \dot{\hat{\eta}}^{1}=f^{1}\left(u, \widehat{\eta}_{1}^{i}, \widehat{\eta}_{2}^{i}\right)+g^{1}\left(u, \widehat{\eta}_{1}^{i}\right)-C_{\lambda_{1}+1}^{1} \theta^{\delta_{1}}\left(\widehat{\eta}^{1}-x^{1}\right) \\
& \dot{\hat{\eta}}^{\lambda_{1}-1}=f^{\lambda_{1}-1}\left(u, \hat{\eta}^{1}, \ldots, \hat{\eta}^{\lambda_{1}}\right)+g^{\lambda_{1}-1}\left(u, \hat{\eta}^{1}, \ldots, \hat{\eta}^{\lambda_{1}-1}\right)-C_{\lambda_{1}+1}^{\lambda_{1}-1} \theta^{\left(\lambda_{1}-1\right) \delta_{1}} \Lambda_{1, \lambda_{1}-1}{ }^{+}(u, \widehat{\eta})\left(\hat{\eta}^{1}-x^{1}\right) \\
& \dot{\hat{\eta}}^{\lambda_{1}}=f^{\lambda_{1}}\left(u, \widehat{\eta}^{1}, \ldots, \hat{\eta}^{\lambda_{1}+1}\right)+g^{\lambda_{1}}\left(u, \widehat{\eta}^{1}, \ldots, \widehat{\eta}^{\lambda_{1}}, \widehat{v}^{1}\right)-C_{\lambda_{1}+1}^{\lambda_{1}} \theta^{\lambda_{1} \delta_{1}} \Lambda_{1, \lambda_{1}}{ }^{+}(u, \widehat{\eta})\left(\widehat{\eta}^{1}-x^{1}\right) \\
& \left(\begin{array}{c}
\dot{\hat{\eta}}^{\lambda_{1}+1} \\
\dot{\hat{v}}^{1}
\end{array}\right)=\left(\begin{array}{c}
f^{\lambda_{1}+1}\left(u, \hat{\eta}^{1}, \hat{\eta}^{2}, \ldots, \hat{\eta}^{\lambda_{1}+1}, x^{\lambda_{1}+2}\right)+g^{\lambda_{1}+1}\left(u, \widehat{\eta}^{1}, \widehat{\eta}^{2}, \ldots, \widehat{\eta}^{\lambda_{1}+1}, \widehat{v}^{1}\right) \\
0
\end{array}\right) \\
& -C_{\lambda_{1}+1}^{\lambda_{1}+1} \theta^{\left(\lambda_{1}+1\right) \delta_{1}} \Lambda_{1, \lambda_{1}+1}^{+}\left(u, \hat{\eta}^{i}, \widehat{v}^{1}\right)\left(\widehat{\eta}^{1}-x^{1}\right) \\
& \dot{\hat{x}}^{1}=f^{1}\left(u, x^{1}, \hat{x}^{2}\right)+g^{1}\left(u, x^{1}\right)-C_{q}^{1} \theta\left(\hat{x}^{1}-x^{1}\right) \\
& \dot{\hat{x}}^{\lambda_{1}-1}=f^{\lambda_{1}-1}\left(u, x^{1}, \ldots, \widehat{x}^{\lambda_{1}}\right)+g^{\lambda_{1}-1}\left(u, x^{1}, \ldots, \widehat{x}^{\lambda_{1}-1}\right)-C_{q}^{\lambda_{1}-1} \theta^{\left(\lambda_{1}-1\right)} \Lambda_{2, \lambda_{1}-1}{ }^{+}(u, \widehat{x})\left(\widehat{x}^{1}-x^{1}\right) \\
& \dot{\hat{x}}^{\lambda_{1}}=f^{\lambda_{1}}\left(u, x^{1}, \ldots, \hat{x}^{\lambda_{1}+1}\right)+g^{\lambda_{1}}\left(u, x^{1}, \ldots, \widehat{x}^{\lambda_{1}}, \hat{v}^{1}\right)-C_{q}^{\lambda_{1}} \theta^{\lambda_{1}} \Lambda_{2, \lambda_{1}}{ }^{+}(u, \widehat{x})\left(\widehat{x}^{1}-x^{1}\right) \\
& \dot{\hat{x}}^{q-1}=f^{q-1}\left(u, x^{1}, \ldots, \widehat{x}^{q}\right)+g^{q-1}\left(u, x^{1}, \ldots, \widehat{x}^{q-1}, \widehat{v}^{1}\right)-C_{q}^{q-1} \theta^{(q-1)} \Lambda_{2, q-1}{ }^{+}(u, \widehat{x})\left(\hat{x}^{1}-x^{1}\right) \\
& \dot{\hat{x}}^{q}=f^{q}\left(u, x^{1}, \ldots, \widehat{x}^{q}\right)+g^{q}\left(u, x^{1}, \ldots, \widehat{x}^{q}, \widehat{v}^{1}\right)-C_{q}^{q} \theta^{q} \Lambda_{2, q}{ }^{+}(u, \widehat{x})\left(\widehat{x}^{1}-x^{1}\right)
\end{aligned}
$$

with

$$
\left.\prod_{k=1}^{q-1} \frac{\partial f^{k}}{\partial x^{k+1}}(u, x)\right)
$$

$$
\begin{aligned}
& F^{\lambda_{1}}(u, x, v)=\left(\frac{\partial f^{\lambda_{1}}}{\partial x^{\lambda_{1}+1}}(u, x) \frac{\partial g^{\lambda_{1}}}{\partial v^{1}}(u, x, v)\right) \\
& \Lambda_{1}(u, x, v)=\operatorname{diag}\left(I_{p}, \frac{\partial f^{1}}{\partial x^{2}}(u, x), \ldots,\right. \\
& \left.\prod_{k=1}^{\lambda_{1}-1} \frac{\partial f^{k}}{\partial x^{k+1}}(u, x), \prod_{k=1}^{\lambda_{1}-1} \frac{\partial f^{k}}{\partial x^{k+1}}(u, x) F^{\lambda_{1}}(u, x, v)\right) \\
& \Lambda_{2}(u, x, v)=\operatorname{diag}\left(I_{p}, \frac{\partial f^{1}}{\partial x^{2}}(u, x), \ldots,\right.
\end{aligned}
$$

\section{Application of UIO to ASP}

4.1. Observer Synthesis. The bioprocess belongs to the nonlinear class of system (6) with $p=2, \lambda_{1}=r=q=2 . X_{s_{i n}}$ and $X_{\mathrm{NH}_{4, n}}$ are the UIs. The measured states are $X_{\mathrm{O}_{2}}$ and $X_{\mathrm{NO}_{3}}$. The UIO diagram is given by Figure 3 , in fact, our objective is to reconstruct the nonmeasured states $X_{s}$ and $X_{N_{4}}$ and the UIs. We suppose that the states vector $x=\left(\begin{array}{l}x^{1} \\ x^{2}\end{array}\right) \in \mathbb{R}^{4}$, the 


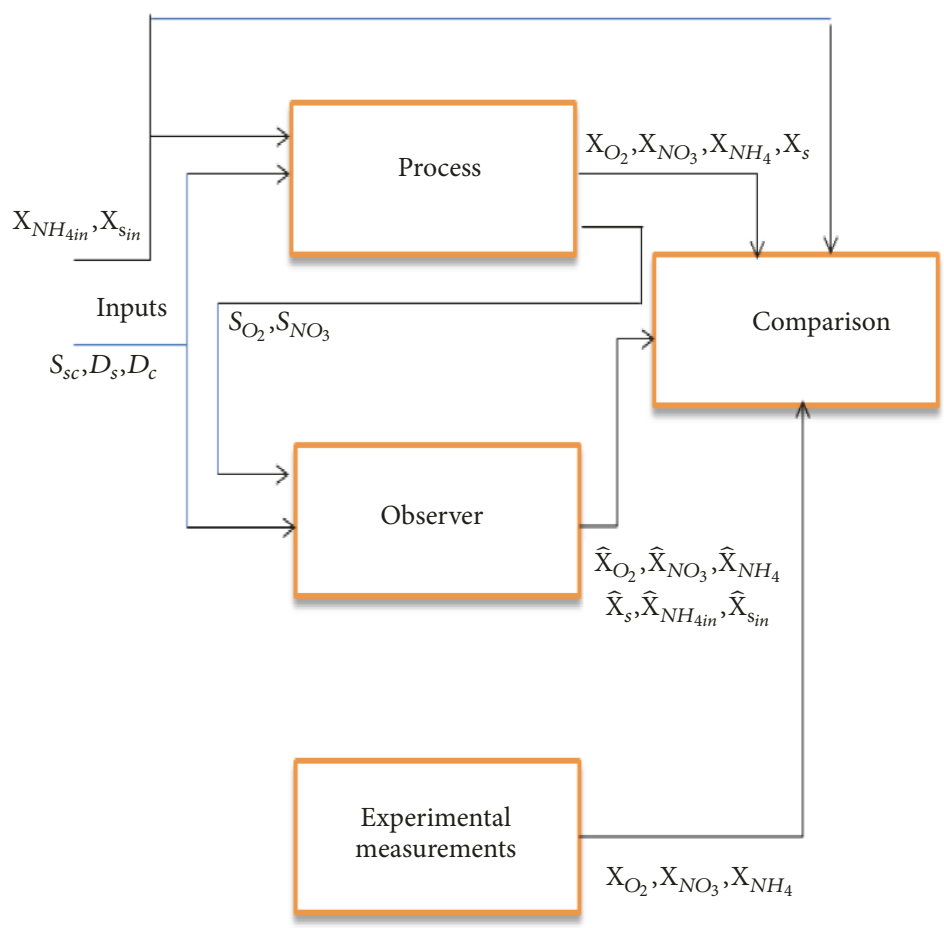

FIgURE 3: UIO diagram.

measured states $x^{1}=\left(\begin{array}{c}X_{\mathrm{O}_{2}} \\ X_{\mathrm{NO}_{3}}\end{array}\right) \in \mathbb{R}^{2}$, and the nonmeasured states $x^{2}=\left(\begin{array}{c}X_{N_{4}} \\ X_{s}\end{array}\right) \in \mathbb{R}^{2} \cdot v=\left(\begin{array}{c}X_{N H_{4} \text { in }} \\ X_{\text {sin }}\end{array}\right) \in \mathbb{R}^{2}$ is the UIs vector and $u=S_{S c} \in \mathbb{R}$ is the known input.

The observer is given by system (34). So the appropriate

$$
\left(\begin{array}{c}
\dot{\bar{X}}_{\mathrm{O}_{2}} \\
\dot{\bar{X}}_{\mathrm{NO}_{3}}
\end{array}\right)=f^{1}\left(u, \hat{x}^{1}, \hat{x}^{2}\right)+g^{1}\left(u, \hat{x}^{1}, \hat{x}^{2}\right)-3 \theta e
$$

$$
\begin{aligned}
\left(\begin{array}{c}
\dot{\hat{X}}_{N_{4}} \\
\dot{\bar{X}}_{S}
\end{array}\right)= & f^{2}\left(u, \hat{x}^{1}, \hat{x}^{2}\right)+g^{2}\left(u, \hat{x}^{1}, \hat{x}^{2}, v\right) \\
& -3 \theta^{2} \Lambda_{1,2}^{+} e \\
\left(\begin{array}{c}
\dot{\hat{X}}_{N H_{4} i n} \\
\dot{\bar{X}}_{S_{i n}}
\end{array}\right)= & -\theta^{3} \Lambda_{1,3}^{+} e
\end{aligned}
$$

with

$$
\begin{aligned}
f^{1}\left(u, x^{1}, x^{2}\right) & =\left(\begin{array}{c}
-\left(D_{s}+D_{c}\right) X_{\mathrm{O}_{2}}+K_{\mathrm{La}}\left(S_{\mathrm{O}_{2} s a t}-X_{\mathrm{O}_{2}}\right)-\frac{1-Y_{H}}{Y_{H}} \beta_{1}-4,57 \beta_{3} \\
-\left(D_{s}+D_{c}\right) X_{\mathrm{NO}_{3}}-\frac{1-Y_{H}}{2,86 Y_{H}} \beta_{2}+\beta_{3}
\end{array}\right) \\
f^{2}\left(u, x^{1}, x^{2}\right) & =\left(\begin{array}{c}
-\left(D_{s}+D_{c}\right) X_{N_{4}}-i_{N B M}\left(\beta_{1}+\beta_{2}\right)-\beta_{3}+\beta_{4} \\
+D_{s} S_{S c}-\left(D_{s}+D_{c}\right) X_{s}-\frac{1}{Y_{H}}\left(\beta_{1}+\beta_{2}\right)+\beta_{5}
\end{array}\right) \\
g^{1}\left(u, x^{1}, x^{2}\right) & =0 ; \\
g^{2}\left(u, x^{1}, x^{2}, v\right) & =\left(\begin{array}{c}
D_{s} X_{\mathrm{NH}_{4} i n} \\
D_{s} X_{s i n}
\end{array}\right) \\
e & =\left(\begin{array}{c}
\widehat{X}_{\mathrm{O}_{2}}-X_{\mathrm{O}_{2}} \\
\widehat{X}_{\mathrm{NO}_{3}}-X_{\mathrm{NO}_{3}}
\end{array}\right)
\end{aligned}
$$


and

$$
\begin{aligned}
& \Lambda_{1,2}=\left[\frac{\partial f^{1}}{\partial\left(X_{N H_{4}}\right)} \frac{\partial f^{1}}{\partial\left(X_{s}\right)}\right] \\
& \Lambda_{1,3}=\Lambda_{1,2}\left[\frac{\partial g^{2}}{\partial\left(X_{N H_{\text {in }}}\right)} \frac{\partial g^{2}}{\partial\left(X_{s_{i n}}\right)}\right] \\
& \frac{\partial f^{1}}{\partial\left(X_{N H_{4}}\right)} \\
& =\left(\begin{array}{c}
-4,57 \alpha_{2} \frac{X_{\mathrm{O}_{2}}}{X_{\mathrm{O}_{2}}+K_{\mathrm{O}_{2 a u t}}} \frac{K_{N H_{\text {taut }}}}{\left(X_{N_{4}}+K_{N H_{4 a u t}}\right)^{2}} \\
\alpha_{2} \frac{X_{\mathrm{O}_{2}}}{X_{\mathrm{O}_{2}}+K_{\mathrm{O}_{\text {2aut }}}} \frac{K_{N H_{\text {taut }}}}{\left(X_{N_{4}}+K_{N H_{\text {taut }}}\right)^{2}}
\end{array}\right) \\
& \frac{\partial f^{1}}{\partial\left(X_{s}\right)}
\end{aligned}
$$$$
=\left(\begin{array}{c}
-\frac{1-Y_{H}}{Y_{H}} \alpha_{1} \frac{X_{\mathrm{O}_{2}}}{X_{\mathrm{O}_{2}}+K_{\mathrm{O}_{2} \mathrm{H}}} \\
-\frac{1-Y_{\mathrm{H}}}{2,86 Y_{\mathrm{H}}} \alpha_{1} \frac{X_{\mathrm{NO}_{3}}}{X_{\mathrm{NO}_{3}}+K_{\mathrm{NO}_{3}}} \frac{K_{\mathrm{O}_{2}}+K_{\mathrm{O}_{2} \mathrm{H}}}{X_{2}}
\end{array}\right)
$$

and

$$
\begin{gathered}
\frac{\partial g^{2}}{\partial\left(X_{N H_{4} i n}\right)}=\left(\begin{array}{c}
D_{s} \\
0
\end{array}\right) ; \\
\frac{\partial g^{2}}{\partial\left(X_{s_{i n}}\right)}=\left(\begin{array}{c}
0 \\
D_{s}
\end{array}\right)
\end{gathered}
$$

4.2. Validation of the UIO with Experimental Data. We have taken advantage of the experiment done during the modeling and identification of the pilot unit [33] to highlight the performance of the studied observer.

The states issued from the process simulation model are compared to the estimated states issued from our observer model as well as their measured values (Figures 4 and 5). The real data (measured) was carried out for the ASWWTP over 6 hours and with a sampling period of 20 minutes [33]. In fact polluted water which comes from sanitary network in the city of Toulouse (France) feeds continuously the pilot unit in order to purify it. We obtained the measurement by taking samples every 20 minutes and analyze them in a biological laboratory to extract the three concentrations (oxygen, nitrate, and ammonia).

The real curves of the UIs are compared with their estimated ones. It is clear that the corresponding curves are almost superimposed. In addition, the UIs (the influent ammonia concentration $X_{N_{\text {Ain }}}$ and the influent substrate concentration $X_{s_{i n}}$ ) are perfectly reconstructed by the UIO despite their variation. We have chosen a variable profile of the UIs to highlight the effectiveness of the UIO. Indeed, the proposed scenario reflects what is really happening in open-air treatment plants. For example, the increase in
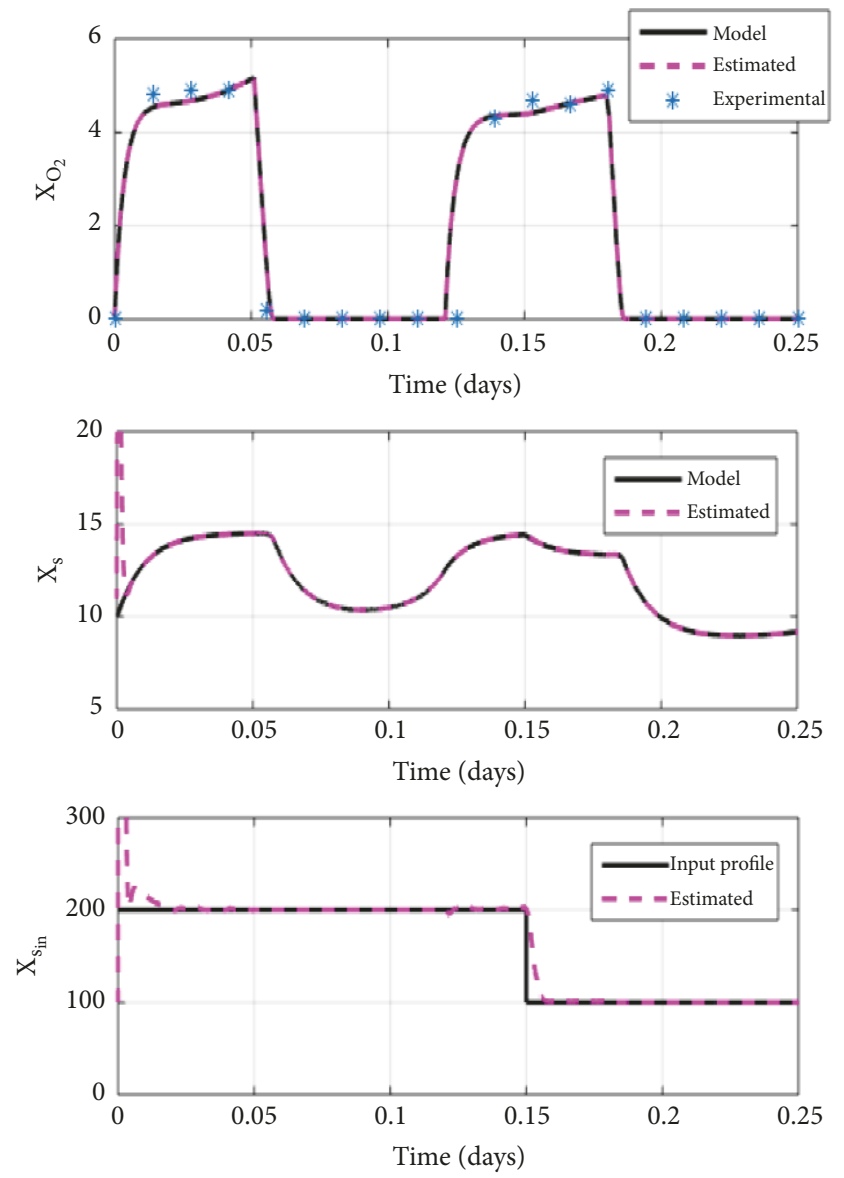

FIGURE 4: Estimation of dissolved oxygen, biodegradable substrate, and the influent biodegradable substrate concentration.

TABLE 4: Error quantification.

\begin{tabular}{lccc}
\hline Error & $X_{\mathrm{O}_{2}}$ & $X_{\mathrm{NO}_{3}}$ & $X_{\mathrm{NH}_{4}}$ \\
\hline $\boldsymbol{e}_{\max }$ & 0.268 & 1.29 & 0.24 \\
$\boldsymbol{e}_{\max \text { relative }}(\%)$ & 5.42 & 19.28 & 4.29 \\
\hline
\end{tabular}

concentration of the "influent ammonia" is due to the arrival of water loaded with heavy mass caused by industrial discharges, while decreasing in concentration of the "influential biodegradable substrate" is due to important (continuous) reversals of rain. The found results evidently show a thorough compromise between estimated and real curves. Moreover, we can show that the state variables signals of the model and the observer are rallied with the experimental measurements. This is quantified by the relative error (Table 4 ). The unknown inputs estimation error converges to zero. The advantages of such observer are that it is relatively simple to design and it has only one adjustment parameter.

\section{Conclusion}

A large class of nonlinear MIMO systems that involves UIs has been studied in this paper in order to synthesize UIO. This observer permits jointly estimating UIs and state variables. 

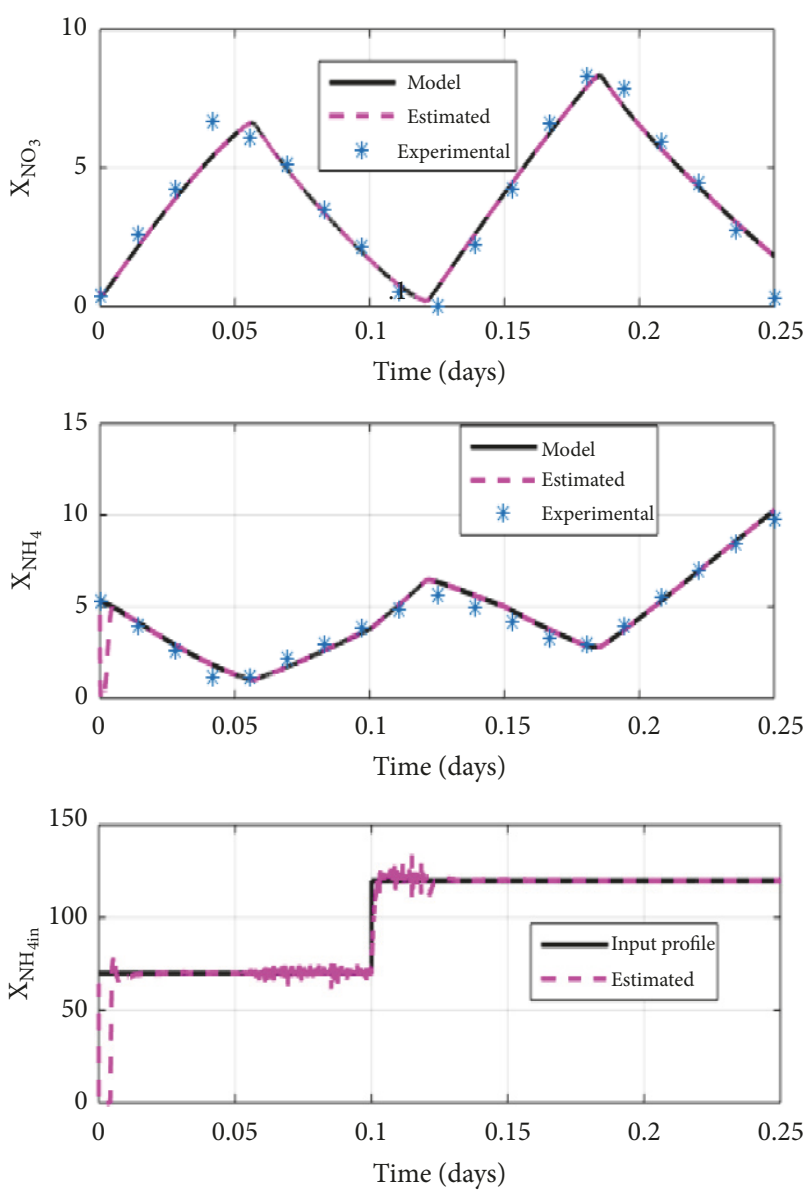

FIGURE 5: Estimation of nitrate, ammonia, and the influent ammonia concentration.

We have applied it to one complicated biochemical ASP. The latter is a reduced activated sludge model having switching anoxic and aerobic phases. By using only two measurements, we have achieved the reconstruction of two state variables $\left(X_{s}\right.$ and $\left.X_{{N H_{4}}_{4}}\right)$ and two UI signals $\left(X_{N_{4 i n}}\right.$ and $\left.X_{\text {sin }}\right)$. Indeed, the obtained simulation results demonstrate quite estimation performances of the suggested observer compared to experimental data. Such observer should be combined with a state control law to ensure that the nitrogen concentration at the settler output does not exceed the standard European norm.

\section{Data Availability}

The data supporting this study are from previously reported studies, which have been cited.

\section{Conflicts of Interest}

The authors declare that they have no conflicts of interest.

\section{References}

[1] M. Henze, C. P. L. Grady, G. V. R. Marais, and T. Matsuo, "Activated sludge model no. 1," IAWQ Scientific and Technical Report 1, London, UK, 1987.
[2] M. Henze, W. Gujer, T. Mino, T. Matsuo, M. C. M. Wentzel, and G. V. R. Marais, "Activated sludge model no. 2," IAWQ Scientific and Technical Report 3, London, UK, 1995.

[3] M. Henze, W. Gujer, T. Mino et al., "Activated sludge model No. 2D, ASM2D," Water Science and Technology, vol. 39, no. 1, pp. 165-182, 1999.

[4] W. Gujer, M. Henze, T. Mino, and M. Van Loosdrecht, "Activated Sludge Model No. 3," Water Science and Technology, vol. 39, no. 1, pp. 183-193, 1999.

[5] U. Jeppsson and G. Olsson, "Reduced order models for on-line parameter identification of the activated sludge process," Water Science and Technology, vol. 28, no. 11-12, pp. 173-183, 1993.

[6] M. A. Steffens, P. A. Lant, and R. B. Newell, "A systematic approach for reducing complex biological wastewater treatment models," Water Research, vol. 31, no. 3, pp. 590-606, 1997.

[7] C. Gómez-Quintero, I. Queinnec, and J. Babary, "A reduced nonlinear model of an activated sludge process," IFAC Proceedings Volumes, vol. 33, no. 10, pp. 1001-1006, 2000.

[8] B. Chachuat, N. Roche, and M. A. Latifi, "Reduction of the asml model for optimal control of small-size activated sludge treatment plants," Journal of Water Science, vol. 16, no. 1, pp. 0526, 2003.

[9] I. Y. Smets, J. V. Haegebaert, R. Carrette, and J. F. Van Impe, "Linearization of the activated sludge model ASM1 for fast and reliable predictions," Water Research, vol. 37, no. 8, pp. 1831-1851, 2003.

[10] M. Mulas, S. Tronci, and R. Baratti, "Development of a 4measurable states activated sludge process model deduced from the ASM1," IFAC Proceedings Volumes, vol. 40, no. 5, pp. 213-218, 2007.

[11] R. Marquez, M. Belandria-Carvajal, C. Gómez-Quintero, and M. Ríos-Bolívar, "Average modeling of an alternating aerated activated sludge process for nitrogen removal," IFAC Proceedings Volumes, vol. 44, no. 1, pp. 14195-14200, 2011.

[12] M. B. Khan, H. Nisar, C. A. Ng, P. K. Lo, and V. V. Yap, "Generalized classification modeling of activated sludge process based on microscopic image analysis," Environmental Technology, vol. 39, no. 1, pp. 24-34, 2017.

[13] S. Caraman, M. Barbu, and E. Ceanga, "Robust multimodel control using qft techniques of a wastewater treatment process," Control Engineering and Applied Informatics, vol. 7, no. 2, pp. 10-17, 2005.

[14] O. Gonzalez-Miranda, M. Rios-Bolivar, and C. GomezQuintero, "Adaptive output feedback regulation of an alternating activated sludge process," in Proceedings of the 2009 European Control Conference (ECC), pp. 424-429, Budapest, Hungary, August 2009.

[15] E. Petre, C. Marin, and D. Seliteanu, "Adaptive control strategies for a class of recycled depollution bioprocesses," Control Engineering and Applied Informatics, vol. 7, no. 2, pp. 25-33, 2005.

[16] C. Vlad, M. Sbarciog, M. Barbu, S. Caraman, and A. Wouwer Vande, "Indirect control of substrate concentration for a wastewater treatment process by dissolved oxygen tracking," Control Engineering and Applied Informatics, vol. 14, no. 1, pp. 38-47, 2012.

[17] C. Foscoliano, S. Del Vigo, M. Mulas, and S. Tronci, "Predictive control of an activated sludge process for long term operation," Chemical Engineering Journal, vol. 304, pp. 1031-1044, 2016.

[18] O. K. Ogidan, C. Kriger, and R. Tzoneva, "Networked control with time delay compensation scheme based on a smith predictor for the activated Sludge Process," Control Engineering and Applied Informatics, vol. 19, no. 3, pp. 79-87, 2017. 
[19] C.-S. Gómez-Quintero and I. Queinnec, "State and disturbance estimation for an alternating activated sludge process," IFAC Proceedings Volumes, vol. 35, no. 1, pp. 447-452, 2002.

[20] D. Dochain, "State and parameter estimation in chemical and biochemical processes: A tutorial," Journal of Process Control, vol. 13, no. 8, pp. 801-818, 2003.

[21] N. R. Chiu, H. J. Navarro, and J. Picó, "A nonlinear observer for bioprocesses using LMI," IFAC Proceedings Volumes, vol. 40, no. 4, pp. 393-398, 2007.

[22] B. Boulkroune, M. Darouach, M. Zasadzinski, S. Gillé, and D. Fiorelli, "A nonlinear observer design for an activated sludge wastewater treatment process," Journal of Process Control, vol. 19, no. 9, pp. 1558-1565, 2009.

[23] M. Triki, M. Farza, and Y. Koubaa, "Unknown inputs observers for a class of non triangular nonlinear systems," in Proceedings of the 15th International Conference on Sciences and Techniques of Automatic Control and Computer Engineering (STA), pp. 259266, Hammamet, Tunisia, December 2014.

[24] H. Hammouri and Z. Tmar, "Unknown input observer for state affine systems: a necessary and sufficient condition," Automatica, vol. 46, no. 2, pp. 271-278, 2010.

[25] K. Mohamed, M. Chadli, and M. Chaabane, "Unknown inputs observer for a class of nonlinear uncertain systems: An LMI approach," International Journal of Automation and Computing, vol. 9, no. 3, pp. 331-336, 2012.

[26] F. J. Bejarano, W. Perruquetti, T. Floquet, and G. Zheng, "Observation of nonlinear differential-algebraic systems with unknown inputs," Institute of Electrical and Electronics Engineers Transactions on Automatic Control, vol. 60, no. 7, pp. 1957-1962, 2015.

[27] K. C. Veluvolu, M. Defoort, and Y. C. Soh, "High-gain observer with sliding mode for nonlinear state estimation and fault reconstruction," Journal of The Franklin Institute, vol. 351, no. 4, pp. 1995-2014, 2014.

[28] M. Farza, M. M’Saad, M. Triki, and T. Maatoug, "High gain observer for a class of non-triangular systems," Systems \& Control Letters, vol. 60, no. 1, pp. 27-35, 2011.

[29] S. H. Saïd, F. M'Sahli, and M. Farza, "Simultaneous state and unknown input reconstruction using cascaded high-gain observers," International Journal of Systems Science, vol. 48, no. 15, pp. 3346-3354, 2017.

[30] B. Boulkroune, M. Darouach, M. Zasadzinski, and S. Gille, "State and unknown input estimation for nonlinear singular systems: application to the reduced model of the activated sludge process," in Proceedings of the Automation (MED 2008), pp. 1399-1404, Ajaccio, France, June 2008.

[31] S. Methnani, J.-P. Gauthier, and F. Lafont, "Sensor fault reconstruction and observability for unknown inputs, with an application to wastewater treatment plants," International Journal of Control, vol. 84, no. 4, pp. 822-833, 2011.

[32] I. Khoja, T. Lahdari, A. Sakly, and F. M'Sahli, "Unknown-input observer for disturbance and state estimation of an activated sludge waste water treatment process," in Proceedings of the 17th International Conference on Sciences and Techniques of Automatic Control and Computer Engineering, STA 2016, pp. 1318, Sousse, Tunisia, December 2016.

[33] C. Gomez-Quintero, Modélisation et estimation robuste pour un procede boues activees en alternance de phases [Ph.D. thesis], Universite Paul Sabatier, Laboratoire dAnalyse et dArchitecture des Syst'emes, Toulouse, France, 2002.
[34] S. Julien, Modélisation et éstimation pour le contrôle d'un procdé boues activées elimiminant l'azote des eaux residuaires urbaines [Ph.D. thesis], ENSEEIHT, UPS Toulouse, France, 1997.

[35] F. Liu, M. Farza, and M. M'Saad, "Non-linear observers for state and unknown inputs estimation," International Journal of Modelling, Identification and Control, vol. 2, no. 1, pp. 33-48, 2007. 


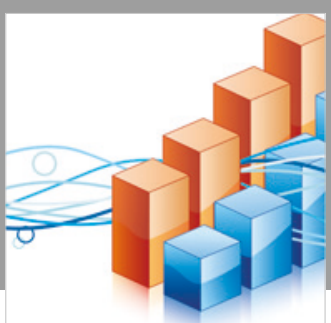

Advances in

Operations Research

\section{-n-m}
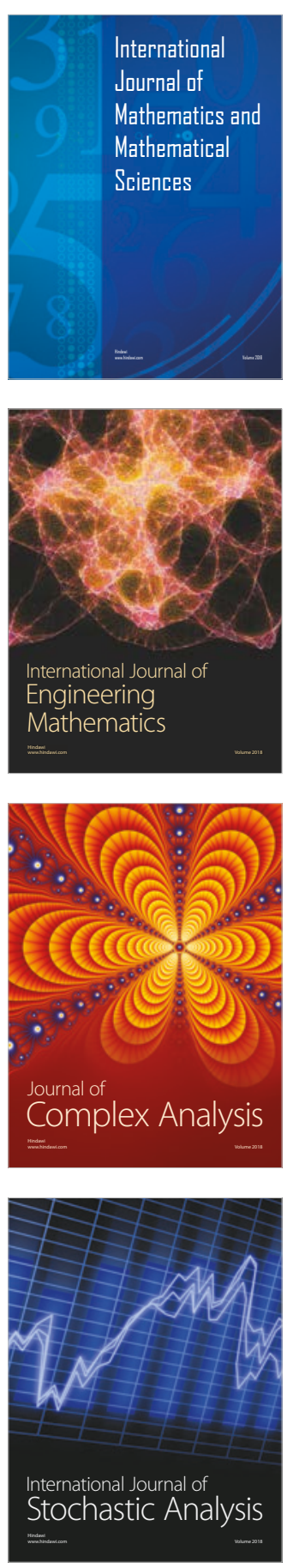
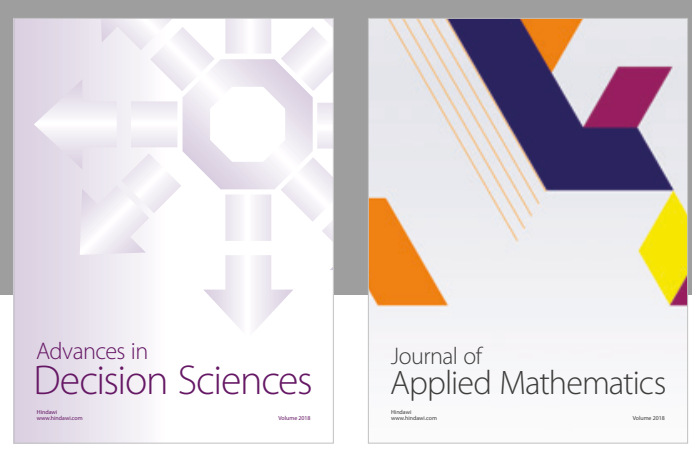

Journal of

Applied Mathematics
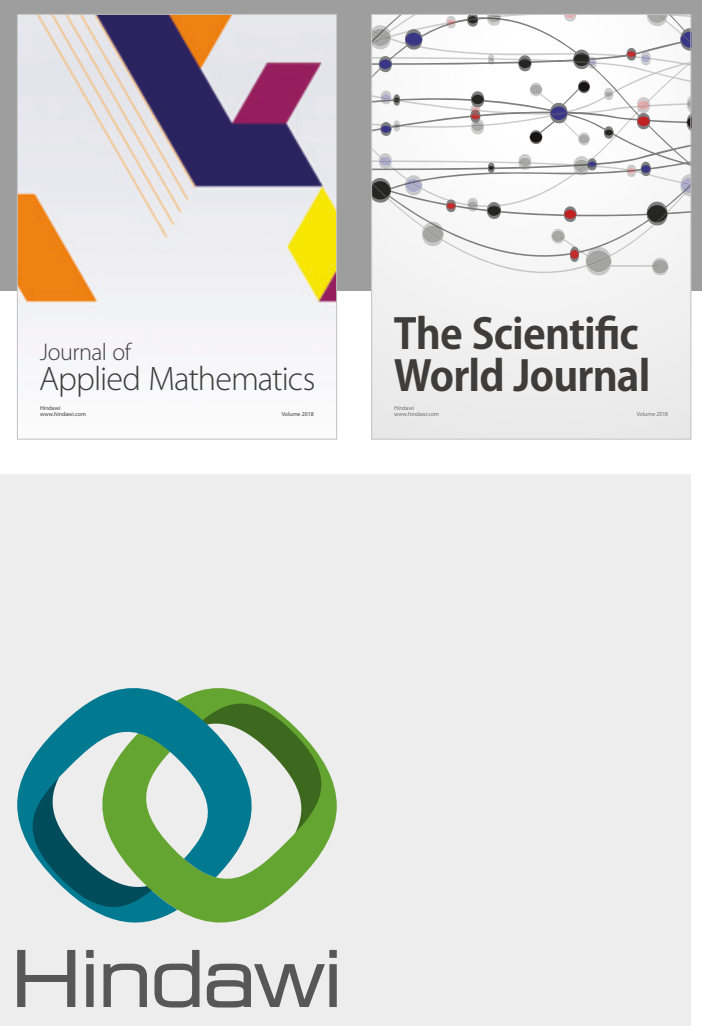

Submit your manuscripts at

www.hindawi.com

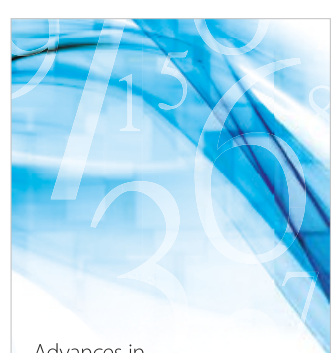

Advances in
Numerical Analysis
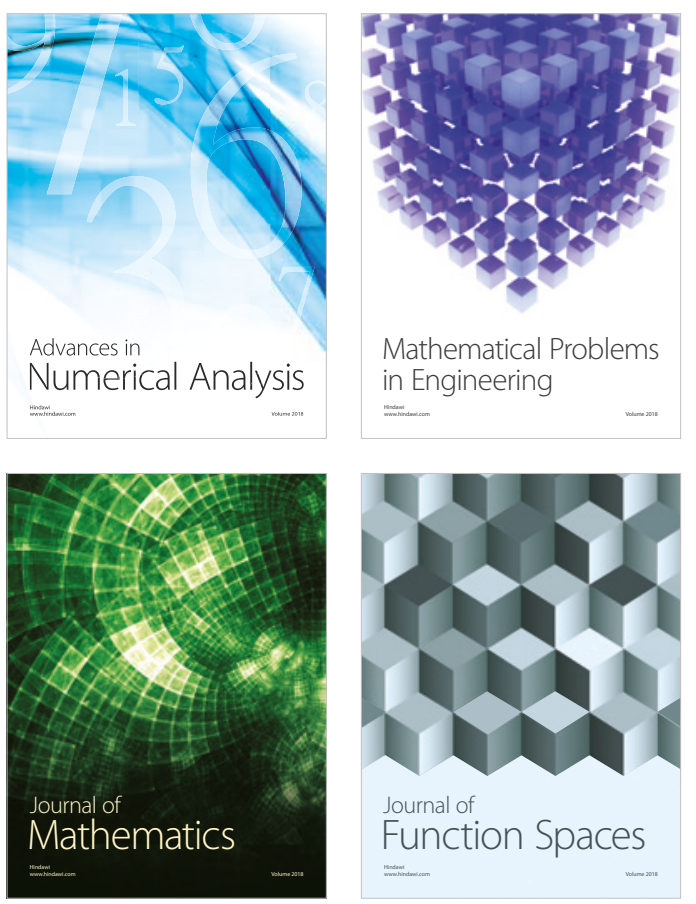

Mathematical Problems in Engineering

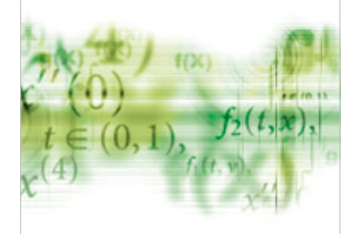

International Journal of

Differential Equations

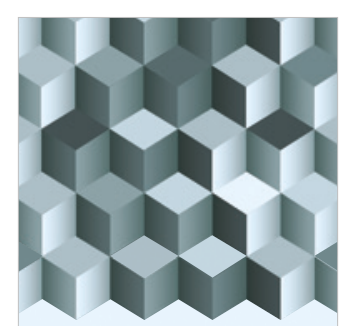

Journal of

Function Spaces

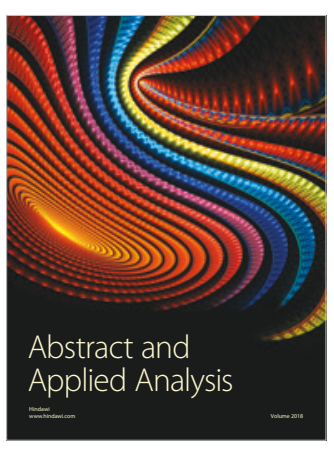

The Scientific

World Journal

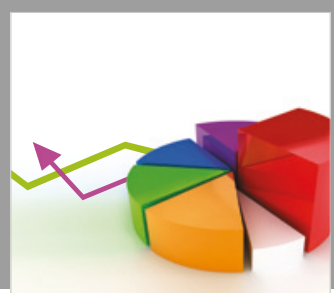

Journal of

Probability and Statistics
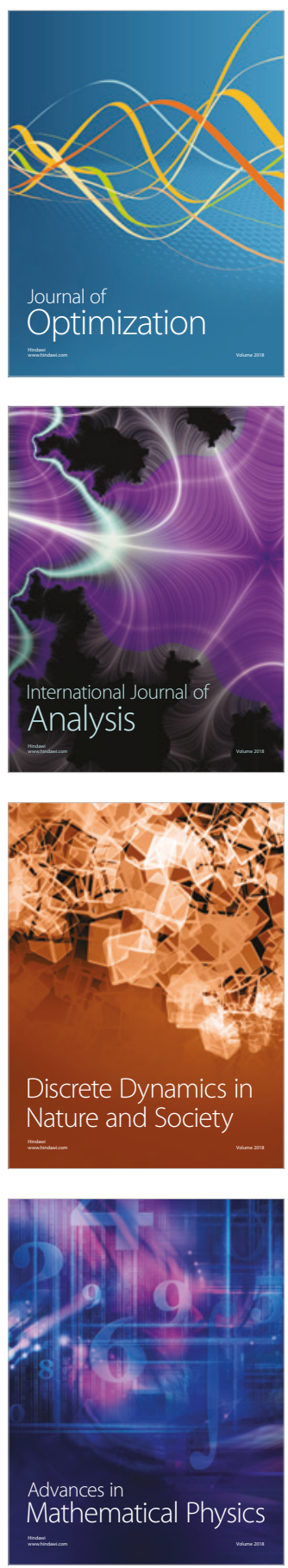